\title{
Igualdad versus Austeridad: Resistencia, protestas y propuestas del movimiento feminista
}

\author{
Begoña SAN JOSÉ SERRAN \\ Fórum de Política Feminista y COMPI ${ }^{1}$
}

Recibido: 01.09.2014

Aceptado: 22.01.2015

\begin{abstract}
RESUMEN:
El neoliberalismo, causante de la crisis financiera iniciada en 2008, gobierna seis años después al $80 \%$ de la población mundial recortando los dos grandes mecanismos de redistribución: el empleo digno y el gasto público social, en un mundo en el que el $8 \%$ de la población acumula el $82 \%$ de la riqueza. El incesante aumento de la desigualdad concita a influyentes intelectuales y moviliza la protesta social. El "colchón familiar", las estrategias familiares de solidaridad y supervivencia frente al paro y los recortes, silencian también nuevas tareas de cuidados y nuevas desigualdades de género. La tenaz salida de las mujeres en búsqueda de empleo hasta 2013 empieza a sucumbir al desánimo en 2014, mientras somos excluidas de la tenue recuperación. El movimiento feminista, revitalizado por una ola de mujeres jóvenes del movimiento $15 \mathrm{M}$, ha realizado sus mayores acciones contra la reforma del aborto, y, con estrategias diversas, aporta la economía feminista a la protesta y la propuesta social. Frente al desmantelamiento de las políticas de igualdad de género y la falta de interlocución política de las organizaciones feministas, el Informe Sombra de las ONGs al Comité para la Eliminación de toda forma de Discriminación de las Mujeres (CEDAW) de Naciones Unidas ha abierto procesos de incidencia política en el 2015, año cargado de expectativas electorales en España y de emplazamientos a nivel mundial para reactivar la agenda Beijing+20 en el marco de los Objetivos de Desarrollo Sostenible.
\end{abstract}

Palabras clave: Neoliberalismo; Economía feminista; Agenda; Interlocución.

\author{
Equality versus Austerity: \\ Resistance, protests and proposals of the feminist movement
}

\begin{abstract}
:
Neoliberalism, cause of the financial crisis in 2008, governs six years later $80 \%$ of the world population by cutting the two major mechanisms of redistribution: worthy jobs and social spending, in a world in which the $8 \%$ the population accumulates $82 \%$ of the wealth. The relentless rise in inequality attracts influential intellectuals and mobilizes social protest. The "family bed", the familiar strategies of solidarity and the survival against unemployment and cuts, also mutes new cares and new inequalities. The tenacious exit of the women in search of employment until 2013 starts succumbing to the discouragement in 2014, while we are excluded from the tenuous recovery. The feminist
\end{abstract}

${ }^{1}$ Coordinadora de Organizaciones de Mujeres para la Participación y la Igualdad 
movement, revitalized by a wave of young women from the $15 \mathrm{M}$ movement, made his major actions against the reform of abortion. By various strategies, the feminist movement incorporates the feminists economics to the social protest. Facing the dismantling of gender equality policies and lack of political discussion of feminist organizations, the NGO Shadow Report to the Committee on the Elimination of All Forms of Discrimination against Women (CEDAW) has opened processes of political incident in 2015; an electoral time in Spain and a time to revive the Schedule Beijing +20 in the context of objetives of the Sustainable Development.

Keywords: Neoliberalism; Feminist Economics; Schedule; Dialogue.

La crisis desencadenada por el estallido en 2008 de la burbuja financierainmobiliaria y su gestión neoliberal en España, pretendidamente de austeridad y estabilidad, han condenado al paro a seis millones de personas, disparado la desigualdad, motivado la desafección hacia las instituciones políticas y generado una amplia movilización social con nuevas formas, en la que ha adquirido un papel central la exigencia de una economía más igualitaria y de mayor control y participación política de la ciudadanía.

En ese cambio radical del contexto económico y político, el movimiento feminista, en su diversidad, ha afrontado nuevos contenidos y formas en la protesta, incorporando una ola de jóvenes activistas y buscando nuevas alianzas. Para hacer política feminista se requieren organizaciones feministas autónomas de los partidos y de otras organizaciones sociales con distintas prioridades, que superen la fragmentación articulándose en torno a reivindicaciones sobre las que interpelar a los poderes instituidos, principalmente los gobiernos, sobre las condiciones de vida de las mujeres. En un tiempo y un país en los que no hay diálogo ni dialéctica entre lo gubernamental y lo no-gubernamental, en la política en general y las políticas de igualdad de género en particular, el cauce de los Informes Sombra de las ONGs al Comité de Naciones Unidas para la Eliminación de toda forma de Discriminación de las Mujeres (CEDAW) abre procesos de incidencia política feminista, cuyo alcance social intento analizar en este artículo. Por otra parte, expongo cómo la economía feminista y su reivindicación de reparto del empleo y de los cuidados de la infancia y las personas en situación de dependencia, pugnan contra el desempleo y los recortes de servicios públicos, además de ser parte de la construcción de abajo a arriba de otra economía y otra política.

\section{LA OFENSIVA NEOLIBERAL AUMENTA LA DESIGUALDAD SOCIAL Y DE GÉNERO, Y LA SILENCIA.}

El neoliberalismo, causante de la crisis de 2008, persiste seis años después en gobernar su solución, no desinflando la burbuja financiera-inmobiliaria, sino la supuesta "burbuja social", debilitando los dos grandes mecanismos de 
redistribución: el empleo digno y el gasto público en educación, sanidad y pensiones, los tres pilares del Estado de Bienestar, así como en el germen de cuarto pilar, los servicios sociales y de atención a la dependencia. Y lo hace no sólo por la fuerza y la represión de la protesta, sino intentando deslegitimarla, silenciando la denuncia y la protesta y tergiversando palabras como "reforma", para hacerla sinónimo de eliminación, "austeridad" como sinónimo de recorte de mecanismos de redistribución, "sostenibilidad" como subordinación de los derechos de las personas a un fatídico poder financiero y "recuperación" primando los indicadores financieros sobre los sociales.

Sin embargo, el Informe del Secretario General de Naciones Unidas sobre Población y Desarrollo considera insostenible e incompatible con la universalidad de los derechos humanos "un mundo en el que el $8 \%$ de la población acumula el $82 \%$ de la riqueza, como parte de una tendencia de acusado aumento de la desigualdad de riqueza en los últimos 20 años"2. Como señalaba Lina Gálvez en el IV Congreso estatal de Economía Feminista en $2013^{3}$, los que gobiernan al $80 \%$ de la población mundial, siguen empecinados en el austericidio. "La austeridad se mantiene como una ideología inmune a los hechos y a la básica refutación empírica (...) una idea muy peligrosa" especialmente para las mujeres, porque aumenta nuestra carga de trabajo no remunerado, forzándonos -ante la falta de corresponsabilidad familiar y social- a asumir estrategias familiares de supervivencia, que reprivatizan y refeminizan las tareas de cuidados, para paliar las estrategias de recortes gubernamentales, y también de las empresariales en determinados sectores; porque la pauta histórica es que tras la crisis el empleo masculino se recupera siempre antes que el femenino, que además sale más precarizado y porque de las crisis se sale con el reforzamiento de los estereotipos tradicionales y de la intersección con otras desigualdades, que hacen retroceder la igualdad de género conseguida anteriormente.

La Unión Europea está en la locomotora del austericidio. La Estrategia Europa 2020, adoptada en plena crisis 2010, se obstina en el fundamentalismo del recorte del déficit público del pacto de estabilidad de 1997. La evaluación

\footnotetext{
${ }^{2}$ Naciones Unidas, Asamblea General, A/69/62 Informe del Secretario General "Marco de medidas para el seguimiento del Programa de Acción de la Conferencia Internacional sobre la Población y el Desarrollo después de 2014”, 12 febrero 2014, epígrafe VI.c. Costo de la desigualdad para el logro del desarrollo sostenible. P. 198.

${ }^{3}$ Lina GÁLVEZ, citando a Ortiz y Cummins, en "Una lectura feminista del austericidio", en Feminismo frente al neoliberalismo, http://www.forumpoliticafeminista.org/neoliberlismoestrategias-y-alianzas-xxxiii-taller/ Fórum de Política Feminista, Madrid, 2014, p. 13.
} 
intermedia por la Comisión Europea en marzo 2014, COM(2014) 130 final $^{4}$, reconoce el fracaso en tres de sus cinco objetivos ${ }^{5}$ :

- La tasa de empleo no avanza hacia el 75\%. De 2010 a 2013 se ha estancado en el $68,4 \%$ en la UE y retrocedido en Grecia del 63,8 al 52,9 y en España del $62,5 \%$ al $58,2 \%$.

- La desigualdad social aumenta. Los ingresos del $20 \%$ de población más rica quintuplican $(5,1)$ los del $20 \%$ más pobre, por el paro, el descenso de salarios y pensiones y la discriminación, alcanzando 7,2 veces en España, donde de 2007 a 2011 se redujo un $42,4 \%$ (de 389 a 224 euros al mes) el ingreso del $10 \%$ más pobre de la población, frente al 5,6\% (de 3.267 a 3.082) del 10\% más rico, según la OCDE.

- La pobreza crece, y afecta a 124 millones de personas en la UE, 10 millones más que en 2010 , y subido del 26,7 al $28 \%$ de la población en España.

El desinterés de la Comisión Europea por el impacto de género de los cambios en el empleo, la desigualdad social, la pobreza, la investigación y la educación se manifiesta en que no desglosa por sexos ni uno solo de los 25 cuadros de datos de dicha evaluación intermedia de los objetivos de la Estrategia 2020, en marzo 2014, pese al llamamiento de la Comisión de Igualdad del Parlamento Europeo ${ }^{6}$. También es clamoroso el silencio de la Unión Europea en el Foro Beijing +20 de los 56 estados de la región ECE (Europa, América del Norte, Israel, Rusia y Asia Occidental) de la ONU en noviembre 2014 en Ginebra, donde, a diferencia de las evaluaciones de 2000, 2005 y 2010, no ha habido Informe de la $\mathrm{UE}^{7}$, y en el semestre transcurrido tras las elecciones europeas y el nombramiento

${ }^{4}$ ec.europa.eu/.../pdf/europe2020stocktaking_es.pdf/

${ }^{5}$ Efectos que previsiblemente no mejorarán sensiblemente con la Iniciativa Europea de Empleo Juvenil de 6.000 millones para 2014-2020 ni el Plan Junckers, los 315.000 millones de euros del "Fondo Europeo para Inversiones Estratégicas" de 2015 a 2018, que no palían la caída del 20\% en inversión pública en la UE de 2008 y 2013, y pueden agravarse con el opaco proyecto de Tratado de Libre Comercio con Estados Unidos, cuyo impacto de género debemos hacer ver.

${ }^{6}$ Parlement Européen, Commission des droits de la femme et de l'égalité des genres. Document de séance A7-0073/2014, 31.1.2014. Rapport sur l'égalité entre les femmes et les hommes au sein de l'Union européenne 2012 (2013/2156(INI)).

${ }^{7}$ El Lobby Europeo de Mujeres, entidad no gubernamental consultiva de la UE, sí ha publicado su Informe 'Beijing20 1995-2015, de las palabras a la acción', en http://www.womenlobby.org/ 
de una Comisaria de Igualdad de Género, que comparte esta tarea con la de Justicia y Consumo, las políticas de igualdad no han vuelto a la agenda política de la UE, y permanecen fuera del tiempo y del espacio en el Instituto Europeo de Igualdad de Género, recluido en Vilnius sin competencias ejecutivas, sino solo de elaboración de informes, estadísticas e indicadores que no se integran en los generales de Eurostat, ni sirven, como establece el Objetivo estratégico H.3 de la Plataforma de Acción de Beijing 1995, para la planificación de políticas públicas y su seguimiento y evaluación por los gobiernos y la sociedad civil.

\section{LA CONVENCIÓN ANTI DISCRIMINACIÓN DE NACIONES UNIDAS $\begin{array}{llllllll}\text { CEDAW } & Y & \text { SUS INSTRUMENTOS DE EVALUACIÓN } & \text { D } & \text { DE }\end{array}$ PARTICIPACIÓN DE LAS ONGS}

Naciones Unidas, como organización de 193 gobiernos, no está inmunizada contra la epidemia neoliberal. Ni contra la burocratización de las instituciones, máxime teniendo una estructura mastodóntica e intrínsecamente alejada de la gente de a pie. Pero el artículo 71 de su Carta fundacional mantiene desde hace setenta años una constante vía de participación de las ONGs, insólita en nuestro país, imprescindible para mantener la legitimación social de su estructura como garante de la universalidad de los derechos humanos y para suplir con activismo voluntario la crónica falta de recursos de la ONU. A ella se añade la evaluación periódica por personas expertas independientes de que gozan algunas de las convenciones de Naciones Unidas, como la CEDAW (Convención para la Eliminación de toda forma de Discriminación de la Mujer). La CEDAW no sólo "declara" a nivel mundial el derecho a la igualdad real entre mujeres y hombres, sino que afirma que aún no se ha alcanzado en ningún lugar del mundo, por lo que obliga a los gobiernos de los 187 países firmantes a realizar actuaciones para eliminar realmente cada forma de discriminación, conforme a la medida 203 de la Plataforma de Acción de Beijing con órganos específicos que realicen políticas específicas, y tengan competencias y recursos para incidir en las políticas generales- y que rindan cuentas cada cuatro años, no sólo permitiendo, sino favoreciendo la participación crítica de las ONGs con "Informes Sombra", que contrastan las luces propias de los informes gubernamentales.

Las organizaciones feministas de España estamos poco acostumbradas a esta forma de participación política, a diferencia de otros países. Pese a que la primera evaluación cuatrienal de nuestro país se hizo en 1993, las organizaciones feministas, las más interesadas en la discriminación de las mujeres, no presentaran Informe Sombra hasta la Sexta, en 2008, cuando lo elaboraron diecisiete organizaciones de la Plataforma Impacto de Género Ya. En enero de 2013 nos enteramos por ONGs de desarrollo de que el Ministerio de Asuntos Exteriores y Cooperación (MAEC) abría el proceso de participación en la elaboración del $6^{\circ}-7^{\circ}$ 
Informe sobre la aplicación de la CEDAW en España en el periodo 2008-2013. La Coordinadora de Organizaciones de Mujeres para la Participación y la Igualdad (COMPI) pedimos que fuera el Instituto de la Mujer, como organismo gubernamental específico para la eliminación de la discriminación de las mujeres, quien coordinara el Informe en vez del MAEC. Paralelamente constituimos una plataforma de unas cincuenta organizaciones, la mitad feministas y la otra mitad de cooperación internacional, y formamos seis grupos de trabajo sobre economía y empleo, violencia de género y trata, educación y cultura, salud, participación política y cooperación internacional, con puestas en común mensuales en el local de Barquillo 44, sede histórica del movimiento feminista en Madrid, hoy trasladada a Bravo Murillo 4, y participación por skype de compañeras de Barcelona, Málaga, Mallorca, San Sebastián y Tenerife. Decidimos que las organizaciones de mujeres inmigrantes, rurales, gitanas y con diversidad funcional no trabajaran aparte, sino transversalizando sus aportaciones en todos los temas.

El 22 de febrero de 2014 convocamos un Encuentro Estatal, para asegurar la representatividad (el 90\% de las 3.000 asociaciones de mujeres en España son locales o autonómicas) y el seguimiento de la aplicación de la Convención también por las Comunidades Autónomas, que gestionan el $40 \%$ de los recursos públicos. Incidimos en ello ya que al Comité CEDAW, en sus Observaciones finales a España (CEDAW/C/ESP/6) de 07.08.2009, señala en el punto 13 que "le preocupa que se le haya facilitado información limitada sobre los mecanismos y estructuras vigentes en los planos provincial y municipal y en las comunidades autónomas", y en el punto 10 "destaca que la Convención es vinculante para todos los poderes públicos e invita al Estado parte a que aliente a sus parlamentos nacionales y autónomos, y a sus cámaras provinciales y municipales" a aplicarla e informar de cómo la aplican. Participamos cerca de doscientas representantes, que debatimos el primer borrador consolidado de Informe Sombra estatal y los Informes Sombra autonómicos de Andalucía, Baleares, Canarias, Cataluña, Castilla y León, Madrid, Murcia, Navarra y País Vasco, que enriquecieron mucho la información y el Informe en su territorio $\mathrm{y}$ a nivel estatal. Abrimos el blog https://cedawsombraesp.wordpress.com/ en el que puede verse el Informe y la relación actualizada de ONGs firmantes, que ha contribuido decisivamente a difundirlo y aumentar su respaldo social, sumando la firma de 277 ONGs en noviembre de 2014, cuando dos representantes de nuestra Plataforma lo expusieron en Ginebra en la pre-sesión sobre España del Comité CEDAW. La sesión final, de la que saldrán las Observaciones finales del Comité a España, será en Ginebra en julio de 2015.

Del tronco de dos años de proceso compartido por más de doscientas organizaciones diversas, han surgido centenares de acciones locales muy diversas, pero con el denominador común del análisis -sobre estadísticas públicas, casos o 
datos contrastables- de situaciones de discriminación de las mujeres, de evaluación de la aplicación por los poderes públicos, con la debida diligencia, de cada artículo de la Convención para eliminarlas en el periodo 2008-2013, de personación de plataformas amplias de asociaciones de mujeres u otras organizaciones sociales como testigos de esas situaciones sociales y de demanda de ser oídas por un Comité de personas expertas independientes, como el CEDAW de la ONU. La dinámica de elaboración autónoma de informes de evaluación periódica de las políticas de igualdad politiza a las asociaciones de mujeres, porque pone el foco en la situación social y su transformación, y dignifica nuestra relación con los poderes públicos, sacándola de la vía muerta del "qué hay de lo mío" cuando la subvención es la única forma de relación, o de la subordinación a órdenes del día establecidos unilateralmente por los gobiernos. Las reglas de contenido, extensión y sustentación de los informes sombra y gubernamentales fijados por la Convención, las Recomendaciones Generales del Comité y manuales como el de Alda Facio ${ }^{8}$ son útiles para la articulación de organizaciones, para la difusión social y para la interlocución con los poderes públicos, y puede trascender, revitalizando los consejos de participación de las mujeres que subsisten a los recortes económicos y políticos (los de la Comunidad de Madrid y de Cantabria se eliminaron en 2010) o abriendo vías de incidencia sobre los poderes central y autonómicos e incluso locales.

El Comité CEDAW, además de su función de garantía del derecho colectivo a políticas contra la discriminación, resuelve demandas individuales, en virtud del Protocolo optativo de la Convención acordado en 1999 y ratificado por España en 2001. El 18 de julio de 2014 ha resuelto la denuncia presentada en 2012 por Ángela González Carreño, apoyada por la asociación Women's Link Worldwide, que también participó en el importante Estudio de la Comisión de Igualdad del Consejo del Poder Judicial sobre la aplicación de la Ley Orgánica 3/2007 para la Igualdad Efectiva de Mujeres y Hombres. El Comité ha condenado al Estado español a indemnizar a Ángela González -cuya hija de 7 años fue asesinada por el padre durante una visita, pese a las 30 denuncias y peticiones de que no viera a la niña sin supervisión- y a evitar situaciones similares, tomando "medidas adecuadas y efectivas para que los antecedentes de violencia de género sean tenidos en cuenta al momento de estipular los derechos de custodia de modo que no se ponga en peligro la seguridad de las víctimas de violencia de género, incluidos los hijos"9 antes del 18 de enero de 2015.

\footnotetext{
$8 \mathrm{http} / /$ www.feminicidio.net/articulo/informes-sombra-instrumentos-para-obligar-losestados-cumplir-con-la-cedaw/

${ }^{9}$ CEDAW/C/58/D/47/2012 Comunicación 47/2012, punto 11.i) página 19.
} 


\section{LAS POLÍTICAS DE IGUALDAD EN ESPAÑA 2008-2013}

El Informe Sombra de las 277 ONGs señala que el Estado central ha reducido de 2009 a 2013 un 49\% su presupuesto para políticas de igualdad de género, ha bajado tres rangos su organismo para la igualdad, de Ministerio de Igualdad (2008-2011) a Dirección General en 2012 ${ }^{10}$, y ha estado más de la mitad de su mandato (de enero 2012 a marzo 2014) sin que el Gobierno apruebe el Plan Estratégico de Igualdad entre Mujeres y Hombres al que le obligan el art. 17 de la Ley de Igualdad y la medida 203 de la Plataforma de Acción de Beijing.

Pese a esta denuncia de las ONGs y a la evidencia de que las políticas de igualdad se hacen con tres "p": programas, personas y presupuestos, el recorte del $49 \%$ en el Presupuesto del Estado para igualdad (programa 232B) y del $23 \%$ en Violencia de Género (programa 232C), se asume acríticamente en los Informes oficiales de Impacto de Género de los Presupuestos del Estado de 2009 a 2014.

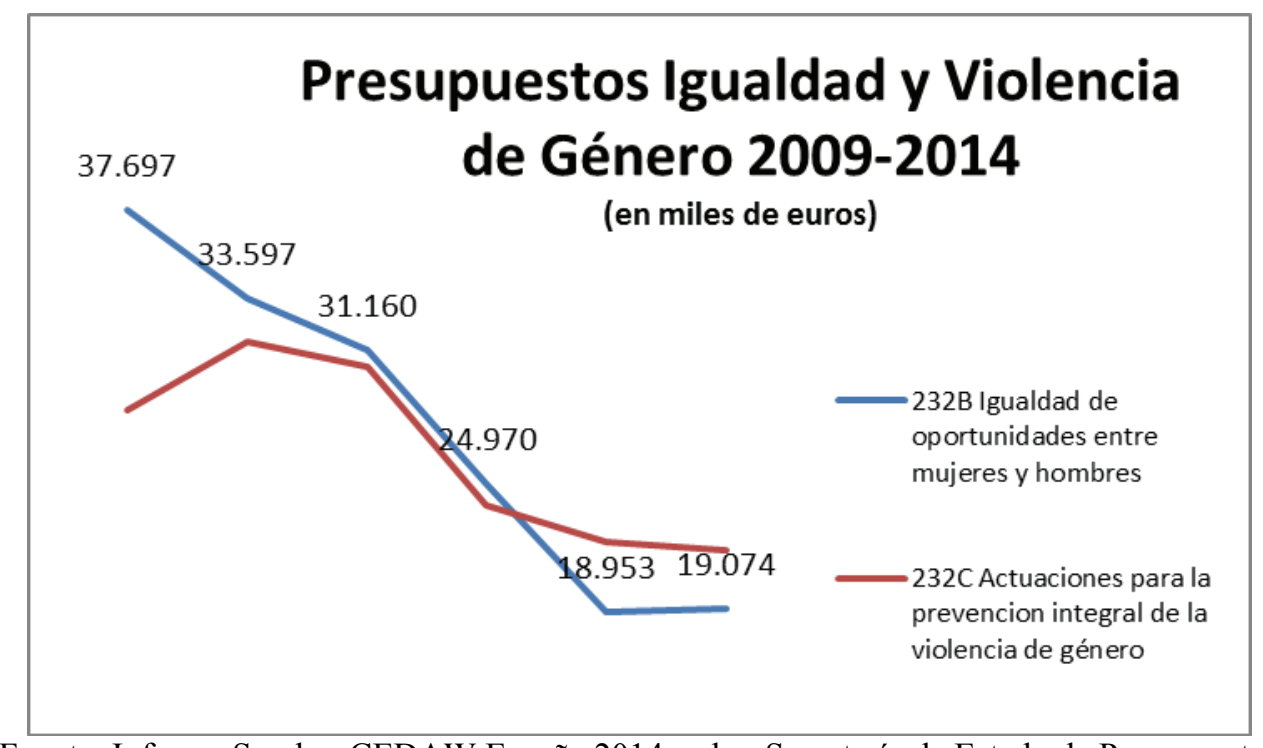

Fuente: Informe Sombra CEDAW España 2014, sobre Secretaría de Estado de Presupuestos

\footnotetext{
${ }^{10}$ Además, el artículo 17 de la Ley 15/2014, de 16 de septiembre, de racionalización del Sector Público y otras medidas de reforma administrativa, denomina al Instituto de la Mujer «Instituto de la Mujer y para la Igualdad de Oportunidades» y amplía sus fines a «la prevención y eliminación de toda clase de discriminación de las personas por razón de nacimiento, sexo, origen racial o étnico, religión o ideología, orientación o identidad sexual, edad, discapacidad o cualquier otra condición o circunstancia personal o social».
} 
Las Comunidades Autónomas también han reducido de 2008 a 2013 un $32,5 \%$ la financiación de sus programas contra la discriminación y la violencia hacia las mujeres, al que destinan un promedio del $0,09 \%$ de su presupuesto. Sus planes languidecen, sus leyes se incumplen y las mujeres en los gobiernos autonómicos han bajado del $39,7 \%$ al $34,2 \%$. Los 4 presididos por mujeres Esperanza Aguirre, Luisa Fernanda Rudí, $\mathrm{M}^{\mathrm{a}}$ Dolores de Cospedal y Yolanda Barcina- paradójicamente bajaron más: Madrid (25\%), Aragón (67\%), Castilla-La Mancha (29\%) y Navarra (40\%). Solo aumentaron Cantabria, Asturias $(62 \%$ mujeres), País Vasco y Melilla

\begin{tabular}{|c|c|c|c|c|c|c|c|c|c|}
\hline \multicolumn{10}{|c|}{$\begin{array}{c}\text { FINANCIACION AUTONOMICA DE PROGRAMAS DE IGUALDAD } \\
\text { DE LAS MUJERES 2008-2013 }\end{array}$} \\
\hline \multirow[t]{2}{*}{ C .Autónoma } & \multirow[t]{2}{*}{ Población } & \multirow{2}{*}{$\begin{array}{l}\text { Organismo de lgualdad } \\
\text { de las Mujeres } \\
2013\end{array}$} & \multicolumn{2}{|c|}{ Presupuesto Igualdad } & \multicolumn{2}{|c|}{ Diferencia 2013-2008 } & \multicolumn{2}{|c|}{ Igualdad 2013} & \multirow{2}{*}{$\begin{array}{l}\text { Total } \\
\mathrm{Pt}^{\circ} \\
€ / \\
\text { habit }\end{array}$} \\
\hline & & & 2008 (euros) & 2013 (euros) & euros & $\%$ & $\begin{array}{l}/ \\
\text { habit }\end{array}$ & \begin{tabular}{|l}
$\% \mathrm{Pt}^{\circ}$ \\
C.A.
\end{tabular} & \\
\hline Andalucía & 8.449 .985 & $\begin{array}{l}\text { Instituto Andaluz de la } \\
\text { Mujer }\end{array}$ & 40.157 .879 & 40.560 .147 & 402.268 & 1,0 & 4,8 & 0,13 & 3.634 \\
\hline Aragón & 1.349 .467 & $\begin{array}{l}\text { Instituto Aragonés de la } \\
\text { Mujer }\end{array}$ & 4.225 .004 & 3.170 .676 & -1.054 .328 & $-25,0$ & 2,3 & 0,06 & 3.785 \\
\hline Asturias & 1.077 .360 & $\begin{array}{l}\text { Instituto Asturiano de la } \\
\text { Mujer y P. Juventud } \\
\end{array}$ & 5.133 .452 & 2.284 .267 & -2.849 .185 & $-55,5$ & 2,1 & 0,06 & 3.530 \\
\hline Balears, Illes & 1.119 .439 & Institut Balear de la Dona & 3.773 .604 & 2.190 .811 & -1.582 .793 & $-41,9$ & 2,0 & 0,06 & 3.214 \\
\hline Canarias & 2.118.344 & $\begin{array}{l}\text { Instituto Canario de } \\
\text { Igualdad } \\
\end{array}$ & 11.513 .123 & 7.018.682 & -4.494 .441 & $-39,0$ & 3,3 & 0,10 & 3.211 \\
\hline Cantabria & 593.861 & $\begin{array}{l}\text { Dirección General de la } \\
\text { Mujer }\end{array}$ & 4.447 .097 & 1.490 .862 & -2.956 .235 & $-66,5$ & 2,5 & 0,07 & 3.862 \\
\hline Castilla y León & 2.546 .078 & $\begin{array}{l}\text { Dirección General de la } \\
\text { Mujer }\end{array}$ & 13.249 .453 & 7.053 .437 & -6.196 .016 & $-46,8$ & 2,8 & 0,07 & 3.724 \\
\hline $\begin{array}{l}\text { Castilla-La } \\
\text { Mancha } \\
\end{array}$ & 2.121.888 & $\begin{array}{l}\text { Instituto de la Mujer de } \\
\text { Castilla-La Mancha }\end{array}$ & 25.967 .200 & 14.107 .310 & -11.859 .890 & $-45,7$ & 6,6 & 0,19 & 3.507 \\
\hline Cataluña & 7.570 .908 & $\begin{array}{l}\text { Institut Català de les } \\
\text { Dones }\end{array}$ & 12.260 .000 & 9.704 .860 & -2.555 .140 & $-20,8$ & 1,3 & 0,03 & 3.962 \\
\hline C. Valenciana & 5.129 .266 & $\begin{array}{l}\text { Dirección General de } \\
\text { Familia y Mujer* } \\
\end{array}$ & 14.573 .970 & 15.278 .250 & 704.280 & 4,8 & 3,0 & 0,12 & 2.538 \\
\hline Extremadura & 1.108.130 & $\begin{array}{l}\text { Instituto de la Mujer de } \\
\text { Extremadura }\end{array}$ & 3.868 .666 & 5.189 .589 & 1.320 .923 & 34,1 & 4,7 & 0,11 & 4.323 \\
\hline Galicia & 2.781 .498 & $\begin{array}{l}\text { Secretaría Xeral } \\
\text { da Igualdade }\end{array}$ & 10.838 .616 & 3.524 .510 & -7.314 .106 & $-67,5$ & 1,3 & 0,04 & 3.408 \\
\hline Madrid & 6.498 .560 & $\begin{array}{l}\text { Dirección General de la } \\
\text { Mujer }\end{array}$ & 44.806 .447 & 22.705 .132 & -22.101 .315 & $-49,3$ & 3,5 & 0,12 & 2.954 \\
\hline Murcia & 1.474 .449 & $\begin{array}{l}\text { DDGG A.Sociales, } \\
\text { Igualdad e Inmigr. y } \\
\text { Violencia* }\end{array}$ & 11.793 .521 & 3.059 .838 & -8.733 .683 & $-74,1$ & 0,8 & 0,07 & 3.036 \\
\hline Navarra & 644.566 & Instituto Navarro para la & 2.794 .533 & 1.807 .286 & -987.247 & $-35,3$ & 2,8 & 0,05 & 5.954 \\
\hline
\end{tabular}




\begin{tabular}{|c|c|c|c|c|c|c|c|c|c|}
\hline & & |familia e igualdad* & & & & & & & \\
\hline País Vasco & 2.193 .093 & $\begin{array}{l}\text { EMAKUNDE - Instituto } \\
\text { Vasco de la Mujer } \\
\end{array}$ & 6.613 .000 & 6.598 .000 & -15.000 & $-0,2$ & 3,0 & 0,06 & 4.776 \\
\hline Rioja, La & 323.609 & & & & & & & 0,00 & 3.786 \\
\hline Ceuta & 84.018 & $\begin{array}{l}\text { Centro Asesor de la } \\
\text { Mujer }\end{array}$ & & 347.120 & & & 4,1 & 0,15 & 2.674 \\
\hline Melilla & 80.802 & $\begin{array}{l}\text { Viceconsejería de la } \\
\text { Mujer }\end{array}$ & & & & & & & \\
\hline TOTAL & $\begin{array}{l}47.265 .32 \\
1\end{array}$ & TOTAL/Media & 216.015 .565 & 145.743 .657 & -70.271 .908 & $-32,5$ & 3,1 & 0,09 & 3.511 \\
\hline
\end{tabular}

Fuente: Informe Sombra http://cedawsombraesp.wordpress.com sobre la web o Boletín Oficial de las Comunidades Autónomas.

*Nota: en la C. Valenciana, Murcia y Navarra el organismo de igualdad suma otras competencias

La Rioja no tiene organismo para la igualdad de las mujeres, sólo el servicio de un Centro Asesor dependiente de la Dirección de Servicios Sociales. En la Comunidad Valenciana el incremento del 4,8\% enmascara la integración de políticas de familias y mujeres. Sólo Andalucía (1\%) y País Vasco (-0,2\%) sostienen presupuestos para políticas visibles de igualdad. Otros datos autonómicos aportados por el Informe Sombra son:

En Murcia el Decreto 17/2010 ha supuesto recortar el 74\% su gasto en igualdad (el Presupuesto General bajó un 12,2\%), suprimir el Instituto de la Mujer creado por su Ley 12/2002, disgregar las competencias de igualdad y contra la Violencia de Género y suprimir los Convenios con los Ayuntamientos para Agentes de Igualdad, despedidas/os en 2011. La Comisión de Igualdad de su Parlamento no se reúne y en su Gobierno el porcentaje de mujeres ha bajado del 23 al 11\%, sólo una.

$(55 \%)$.

- $\quad$ La siguen en recortes Galicia (67\%), Cantabria (66\%) y Asturias

- Castilla y León redujo un 44\% sus presupuestos para igualdad: eliminó en 2011 las transferencias a Planes y Agentes de igualdad de ayuntamientos, a asociaciones de mujeres (excepto la Red Madre, anti-aborto), a mujeres del medio rural y a formación de paradas; cerró en 2012 el Centro de atención 24 horas a maltratadas abierto 2 años antes y 18 oficinas de Red Mujer Empleo.

- En Andalucía la "arquitectura de género" ha mantenido los órganos, aprobado leyes y extendido el Informe de Impacto de Género de sus Presupuestos, publicado desde 2005 con alto nivel político y técnico, desagregando por sexos el $50 \%$ de los datos, pero que no es participativo, pese a la creación del Consejo 
Andaluz de Participación de las Mujeres y el Observatorio de la Violencia de Género.

- En Navarra el Decreto Foral 75/2012 transforma el Instituto de Igualdad en "Instituto para la Familia e Igualdad", que refuerza la tradicional identificación de mujeres con familia, cuidados y políticas de carácter asistencial; ha bajado un $63,85 \%$ el presupuesto para Igualdad y desde 2010 no hay Plan de Igualdad ni Informes de impacto de género. Sólo se ha realizado un estudio, no publicado ni aplicado, de igualdad de género en el marco del Plan para Personas con Discapacidad 2010-2013.

- En Catalunya, junto a la "austeridad" neoliberal, también se ha retrocedido del mainstreaming de equidad de género al asistencialismo: el Institut Català de les Dones ha pasado en 2010 de depender de la Consejería de Presidencia a la de Bienestar y Familia, y reorientado a políticas tradicionales, reducidas y en riesgo de desaparición.

- $\quad$ La Comunidad de Madrid no tiene desde 2005 Plan de Igualdad, y pese a su Ley 5/2005 integral contra la violencia de género, sólo ha adoptado Plan para aplicarla de 2005 a 2008 y suprimió por la Ley 9/2010 el Consejo asesor del Observatorio Regional de la Violencia de Género y el Consejo de la Mujer.

- En las Islas Baleares por el recorte 2008-2013 del 42\% del presupuesto del Institut Balear de la Dona se cierran por las tardes el Centro de Información a Mujeres, haciéndolo inaccesible a estudiantes y trabajadoras, los puntos de información jurídica en pueblos, 9 de las 10 Oficinas de Ayuda a Víctimas del Delito (90\% de usuarias mujeres) e Infosex (información sexual y afectiva a jóvenes). Se han eliminado la Dirección de Igualdad del Consejo Insular de Mallorca y 2 Agentes de igualdad de Palma.

En Canarias se mantienen las transferencias a los Cabildos para la red de atención a víctimas de violencia de género. Pero de 2009 a 2013 la partida para la prevención de la violencia, otras acciones de igualdad y fomento del tejido asociativo feminista, se ha reducido en más de la mitad.

En los Ayuntamientos, la ley 27/2013 de reforma de la Ley de Bases de Régimen Local ha eliminado su artículo 28, que desde 1984 les atribuía la competencia potestativa de promoción de la igualdad de las mujeres, en consonancia con el artículo 9.2 de la Constitución y las Cartas Europeas de Autonomía Local (BOE de 24.02.89) y para la Igualdad de Mujeres y Hombres en la vida local (CMRE 2006). Sólo podrán realizar actuaciones de promoción de las 
mujeres si logran delegación y financiación autonómicas ${ }^{11}$. Los servicios más amenazados son los puntos de atención jurídica, psicológica y social a las víctimas de violencia de género, que por el artículo 19 de la Ley Orgánica 1/2004 de Protección Integral contra la Violencia de Género deben prestarse por convenio de las Comunidades Autónomas y las Corporaciones Locales. Su cierre desactivaría aún más el principal mecanismo de dicha Ley, los juzgados de violencia contra la mujer, manifestado por el descenso de denuncias (17\% de 2008 a 2013, de 142.125 a 124.894), órdenes de protección y condenas, mientras no cesa la sangría de mujeres asesinadas. En resumen, hace siete años, las administraciones central, autonómica y local aprobaban objetivos, programas, presupuestos y órganos para promover la igualdad entre mujeres y hombres y luchar contra la violencia de género, que eran una prioridad política. Hoy triunfa el lema de Esperanza Aguirre "la igualdad igual da" 12 , y las administraciones la abandonan, pretextando, en el mejor de los casos, que la deben hacer otros "para evitar duplicidades" o que es superflua frente a la prioridad absoluta del pago de la deuda.

La reforma del Consejo General del Poder Judicial ha salvado finalmente su Comisión de Igualdad creada en 2007, pero en marzo de 2014 la ha separado de su Observatorio de la Violencia de Género, pese al recién ratificado Convenio del Consejo de Europa (Estambul 2011), que exige unir las políticas de igualdad y las de eliminación de la violencia hacia las mujeres ${ }^{13}$ y ha privado a ambas de retribución, reduciendo por tanto su dedicación y sus Memorias, que antes emitían trimestralmente y eran una referencia institucional y social.

Según las cifras del Informe Sombra las políticas de igualdad representan el 0,005\% del Presupuesto del Estado central, 0,09\% de los autonómicos y 0,24\% de

\footnotetext{
${ }^{11}$ Doce de las CCAA han legislado para eludir la asunción y financiación de competencias municipales, pero también su cierre: Galicia Ley 5/2014, Madrid Ley 1/2014, Cataluña Decreto Ley 4/2014, Murcia Decreto-Ley 1/2014, Andalucía Decreto-Ley 7/2014, Castilla y León Decreto-Ley 1/2014, País Vasco Circular de 11 de marzo de 2014, Asturias Decreto 68/2014, Extremadura Decreto 265/2014, Islas Baleares Decreto Ley 2/2014, La Rioja Ley 2/2014, Aragón Circular 1/2014.

${ }^{12}$ Diario Público, 22.02.2010 http://www.publico.es/espana/aguirre-habria-eliminarministerio-igual.html/

${ }^{13}$ Exige, además, actuaciones para la coordinación entre estados, la cooperación con las ONGs, la recogida y publicidad de datos, educación para la igualdad y contra la violencia en todos los niveles educativos; prohíbe supeditar a la presentación de denuncia los servicios a las víctimas y conceder la custodia y el derecho de visita a hijos e hijas de los maltratadores, $\mathrm{y}$ exige atención especial a migrantes $\mathrm{y}$ asiladas. Texto completo en http://www.boe.es/boe/dias/2014/06/06/pdfs/BOE-A-2014-5947.pdf/
} 
los de los ayuntamientos ${ }^{14}$. Consolidándolos, es decir, restando las transferencias entre las tres administraciones territoriales ${ }^{15}$, son aproximadamente unos 150 millones, el 0,04\% del gasto público y el 0,01\% del PIB, un suelo de gasto público específico sin el cual no hay política de igualdad de género, porque, aun existiendo nominalmente, pierde, en momentos irrecuperables de nuestras vidas individuales y colectivas, la visibilidad social y las herramientas políticas señaladas en la medida 203 de la Plataforma de Acción de Beijing 1995. Esta medida, para el Objetivo estratégico $\mathrm{H}$ sobre mecanismos institucionales para el avance de las mujeres, señala que los gobiernos deben asignar la responsabilidad del adelanto de la mujer a nivel de ministerio, con mandatos y atribuciones claramente definidos, recursos suficientes y funciones de fomento, comunicación, coordinación y vigilancia de la aplicación de la igualdad en los doce objetivos sobre pobreza, educación, salud, violencia, economía, poder y toma de decisiones, derechos humanos, medios de comunicación, medio ambiente y derechos de las niñas.

Los datos del Informe Sombra están sacados de los presupuestos oficiales que usan los gobiernos, pero el gobierno de España contestó en mayo 2014 al cuestionario de la ONU para la elaboración del Informe Beijing+20 a nivel estatal, regiona $^{16}$ y mundial, que el presupuesto para igualdad de género es cien veces mayor: 1.042 millones anuales para igualdad y 390 millones para violencia, basándose no en las partidas presupuestarias, sino en la famosa memoria económica del Plan Estratégico para la Igualdad de Oportunidades 2014-2016, que cifra su presupuesto trienal en 3.126.974.430 euros, y la Estrategia Nacional para la Erradicación de la Violencia sobre las Mujeres 2013-2016 con un presupuesto cuatrienal de 1.558.611.634 euros. Es una falacia que el recorte en las políticas específicas de igualdad de género se compense por una supuesta transversalidad en las políticas generales que nadie controla ni mide. No es de extrañar que el

\footnotetext{
14 “La situación de los Ayuntamientos en España, sus carencias económicas y problemas de gestión: diagnóstico y propuestas en una perspectiva europea” FEMP 2006, cuadro de la P. 75. Estima que el 0,5\% del gasto municipal en igualdad de género se financiaba por el Estado, $17 \%$ por las CC. Autónomas, $0,3 \%$ por las diputaciones, y $0,3 \%$ por otros, como el Fondo Social Europeo.

${ }^{15}$ El gasto público en España se gestiona en un 58\% por el Estado, (incluyendo el 33\% de la Seguridad Social), 30\% por las CC. Autónomas y 12\% por los Ayuntamientos. En 2013 el gasto público no financiero fue de 385.670 millones de euros, un 37,7\% (9 puntos menos que en la Eurozona) del PIB (1.023 miles de millones), mientras la deuda pública ascendió a 958.031 millones, un 93,7\% del PIB.

${ }^{16}$ El Informe 2014 de la región ECE, en la que se integran 56 países, entre ellos la Unión Europea, que no presentó informe conjunto en 2014, está en http://www.unece.org/genderwelcome-new/meetings-and-events/beijing-platform-foraction/inter-governmental-meeting/2014/beijing-20-regional-review-meeting/beijing-20regional-review-meeting.html\#/
} 
importante Informe Beijing+15 de la presidencia sueca de la Unión Europea dijera: "sigue siendo un reto conocer exactamente cuanta financiación comunitaria se gasta en políticas de igualdad entre los sexos" y "una información más detallada sobre esta cuestión de todos los Estados miembros permitiría determinar el peso de las cuestiones de igualdad entre los sexos desde un punto de vista financiero" ${ }^{17}$.

\section{EL EMPLEO FEMENINO EN ESPAÑA 2008-2014: RESISTENCIA, PRECARIEDAD, PERSPECTIVAS.}

El paro es la principal preocupación de la sociedad española, como refleja constantemente el Centro de Investigaciones Sociológicas (CIS). Por ello, su medición oficial, la Encuesta de Población Activa (EPA), tiene un gran impacto mediático, social y político, trimestre a trimestre, y es quizás la estadística cuya difusión crea en mayor medida opinión pública y agenda política. Aunque en 2011, año doblemente electoral, el bombardeo mediático priorizó el dato de la prima de riesgo de nuestra deuda pública, emitido por las empresas estadounidenses Standard \& Poor's, Moody's y Fitch, el PP usó como lema electoral "cinco millones de parados". Los durísimos 18 decretos-leyes de su gobierno en $2012^{18}$ bajaron la prima de riesgo, pero extendieron el paro a seis millones de personas.

En empleo es esencial para la independencia económica de las mujeres, pero accedemos a él, como ampliamente ha explicado la economía feminista, lastradas por la sobrecarga de trabajo no pagado de cuidados familiares, que el concepto de actividad de la EPA -personas de 16 a 65 años con empleo o buscándolo- desprecia de forma sexista, al calificar como Población Económicamente Inactiva a 3,5 millones de mujeres en "labores del hogar". El Informe para Beijing+15 de la Unión Europea en 2010 señalaba que España es el cuarto país, tras Luxemburgo, Malta y Chipre, con mayor proporción de mujeres que no buscan empleo por cuidar a niños, mayores dependientes u otros familiares. Por ello, la Tasa de Actividad Femenina (TAF) en España es muy inferior a la de los hombres y a la de las mujeres en otros países de Europa, alcanzando el 50\%

\footnotetext{
${ }^{17}$ Beijing +15 , la Plataforma de Acción y la Unión Europea, págs 14, 39 a 48 y Anexo III (pgs.168 a 170) de la versión en castellano. Sobre Mecanismos institucionales de igualdad en 2009 sólo da cifras del número de personas asignadas al organismo de igualdad y a si tiene competencias de iniciativa legislativa y política o solo de información y formación. http://www.apramp.org/documento.asp?id=133

18 Ver "Las asociaciones de mujeres frente al empleo, la violencia de género y la participación" 25 años del Fórum de Política Feminista 1987-2012, pág. 52 http://www.forumpoliticafeminista.org/25-a\%C3\%B1os-del-forum-de-pol\%C3\%ADticafeminista-y-de-feminismo/
} 
justo al comenzar esta crisis en $2008^{19}$. Pero durante la crisis las mujeres "inactivas" por dedicarse a "labores del hogar" han bajado un llamativo 32,1\% (de 782.500 a 251.400), mientras, correlativamente, 734.600 mujeres se han incorporado a la población activa (de 9.957.800 en el tercer trimestre de 2008 a 10.627 .800 en el tercer trimestre de 2013), elevando la tasa de activas hasta el $54 \%$ en 2012 , y descendiendo al 53,4\% en el tercer trimestre de 2014. Pese al desempleo de larga duración y al empeoramiento de las condiciones de empleo, las mujeres españolas se resisten, hasta ahora, a "volver al hogar", de manera que el papel de mano de obra de reserva lo están jugando los inmigrantes, de los que en los tres últimos años 570.000 , el 44,3\% mujeres, se han marchado silenciosamente, y los jóvenes ${ }^{20}$. tres etapas:

El impacto de género de la evolución del empleo de 2008 a 2014 ha tenido

de 2008 a 2011, cuando se destruyeron 2,47 millones de empleos, de los que el $80 \%$ fueron de hombres, por la masculinización del empleo en la construcción, el epicentro de la crisis. Se redujo la brecha de género no por mejora del empleo de las mujeres, sino por empeoramiento más rápido del de los hombres.

- de 2012 a 2013, cuando la destrucción del empleo se ralentizó, y se destruyeron 629.800 empleos, de los que el $60 \%$ fueron masculinos.

del tercer trimestre de 2013 al último dato publicado por la EPA (tercer trimestre 2014), en que ha habido una recuperación de 274.000 empleos, que en un $81 \%$ han sido de hombres, lo que confirma la tesis de Lina Gálvez antes apuntada: el empleo público y el empleo femenino serán los más difíciles de recuperar.

La precarización del empleo, por el descenso de los salarios y la protección social y el aumento de los contratos a tiempo parcial, que ya son el $26 \%$ de los femeninos y $8 \%$ de los masculinos, y la temporalidad, que producen el empleo con pobreza (menos de 676 euros al mes) exige que la EPA refleje no sólo la cantidad de empleos, sino su calidad. En este sentido es positivo que el Consejo sobre las Mujeres y la Economía de la Unión Europea de 19.06.14 acordara, aunque con la oposición de España y Hungría, ${ }^{21}$ nuevos indicadores de Beijing sobre el peso del

\footnotetext{
${ }^{19}$ La Tasa de Actividad Femenina en esta crisis duplica la de 1973, en la crisis del petróleo, cuando 311.000 mujeres desanimadas abandonaron la búsqueda de empleo y la tasa de paro de mujeres duplicó la de hombres

20 http://www.ine.es/inebaseDYN/epa30308/docs/evolucion_epa.pdf Análisis de la evolución reciente de la población activa en España (ITr 2011 a ITr 2014) 21

file://C:/Users/HP/Downloads/Mujeres\%20y\%20economia\%2019.06.14\%20UE\%20en.pdf/
} 
empleo a tiempo parcial, su tasa equivalente del tiempo completo, y la relación entre pobreza y violencia contra las mujeres, cuya publicación y difusión deben servir, como señala el Objetivo H.3 de la Plataforma de Acción de Beijing 1995, para la sensibilización social y hacer políticas contra la discriminación.

La cobertura de la protección por desempleo desciende dramáticamente, por los cambios normativos y el paro de larga duración, con un descenso interanual del $11,9 \%$, que supone que solo 1.140 .400 paradas, el $42,4 \%$ de las reconocidas por la EPA y 1.343 .100 parados, el $45,8 \%$, reciben prestación por desempleo, cuya cuantía, en el caso de las contributivas, las más altas, es en promedio de $871 € /$ mes para los hombres y 706 para las mujeres. De ahí la necesidad de que participemos en la reivindicación de la Renta mínima garantizada e individual, para no reproducir la dependencia familiar y la discriminación de las mujeres.

\section{PERSONAS BENEFICIARIAS DE PRESTACIONES POR DESEMPLEO}

(en miles) julio 2014

\begin{tabular}{rrcc} 
jul-14 & \multicolumn{2}{c}{ Variación s/ jul-13 } & Mujeres \\
Personas & Personas & $\%$ & $\%$ \\
$1.018,1$ & $-251,4$ & $-19,8$ & 47,72 \\
$1.189,3$ & $-106,9$ & $-8,2$ & 45,01 \\
262,1 & 26 & 11 & \\
$2.469,4$ & $-332,2$ & $-11,9$ & \\
986,2 & $-103,6$ & $-9,5$ & \\
75,8 & 1,7 & 2,3 & \\
127,3 & $-4,9$ & $-3,7$ & 64,58 \\
$3.658,7$ & -439 & & 45,92
\end{tabular}

http://www.empleo.gob.es/es/estadisticas/resumenweb/rud.pdf/

\section{EL AJUSTE SILENCIOSO CON RE-FAMILIARIZACIÓN Y RE- FEMINIZACIÓN DE LOS CUIDADOS ES INSOSTENIBLE}

El silencio sobre la desigualdad de género se intensifica sobre el "trabajo de cuidados" asignado a las mujeres. El "colchón familiar" al que se alude para

Sobre la postura de España ver pag. 6 del Boletín de Empleo de la Comunidad de Madrid marzo

2014

http://www.madrid.org/cs/Satellite?blobcol=urldata\&blobheader=application\%2Fpdf\&blob

headername $1=$ Content-

Disposition\&blobheadervalue $1=$ filename $\% 3$ DBOLET $\%$ C $3 \% 8$ DN + EPSSCO + ABRIL +2014 . pdf\&blobkey $=$ id\&blobtable $=$ MungoBlobs\&blobwhere $=1352849551016 \&$ ssbinary $=$ true 
explicar que con un $25 \%$ de paro, y más del $50 \%$ entre los menores de 30 años, no haya un estallido social, aparte de ignorar a la población inmigrante, que hace de colchón pero no tiene colchón, acepta acríticamente cambios no deseados ni deseables en los hogares: el retraso en la emancipación de los jóvenes, la falta de una habitación propia, los desahucios, la reagrupación de tres generaciones o de varias familias en una vivienda, el sostenimiento por personas jubiladas de sus familiares en edad laboral, las convivencias forzadas, el descenso en un $21 \%$ del número de matrimonios. En la re-familiarización hay mucha re-feminización, la reasunción de muchos mandatos de género con tensiones físicas, psicológicas y éticas, que también acompañan al descenso de 2008 a 2013 de un $18,1 \%$ en el número de nacimientos, de 1,44 a 1,27 hijos por mujer, de lo que el gobierno no habla mientras invoca sistemáticamente "la demografía" para el endurecimiento de las pensiones, el recorte sanitario o incluso el anteproyecto de recorte del derecho al aborto. A este aumento del trabajo no pagado por la pérdida de ingresos se suma el generado por los recortes en servicios públicos: la pérdida de plazas de educación infantil (13.800 en el curso 2013-2014), el cierre de comedores escolares y de transporte sanitario y escolar, el desmantelamiento de la Ley de atención a la dependencia. Baja el empleo y sube un trabajo que no se cuenta ni cuenta, no entra en el reparto familiar y social.

El Instituto Nacional de Estadística (INE) mide el trabajo doméstico no pagado en un estudio ${ }^{22}$ poco difundido y por tanto inoperante para crear reconocimiento social y políticas públicas de reparto de los cuidados. Este estudio estima que el tiempo dedicado al trabajo doméstico no remunerado en España creció un 4,3\% de 2003 a 2010, que las mujeres lo siguen realizando en sus dos terceras partes, al dedicar más del doble de tiempo que los hombres: 4 horas y 7 minutos diarios, frente a 1 hora y 54 minutos, y que su valor es el $36 \%$ del PIB, unos 343.000 millones de euros, cifra sensiblemente superior, por el valor/hora asignado, a la del 27\% del PIB del mismo INE en 2003 (Angulo y Hernández, 2014, 29-46).

El principal medio de reparto social de la parte socialmente imprescindible de trabajo doméstico - los cuidados a la infancia y a mayores dependientes- es la sustitución de los cuidados familiares no pagados por servicios públicos profesionales, es decir, pagados. La Ley de Dependencia de 2006 planteó la asunción gradual por el Estado (central y autonómico), de los servicios a las

\footnotetext{
${ }^{22}$ Valoración del trabajo doméstico en 2010 y su comparación con la de 2003. Estimación de la serie 2003-2010, de Carlos Angulo y Sara Hernández, págs. 29 a 46 de Otras facetas de la Encuesta de Empleo del Tiempo 2009-2010 aplicando el manual de Eurostat sobre cuentas satélites del trabajo no remunerado en la contabilidad nacional. INE Documentos de trabajo 1/2014.
} 
personas mayores de 3 años que necesitan ayuda para funciones de la vida diaria, que se estiman entre 1,5 y 2,6 millones, hasta alcanzar la cobertura universal en 2015. En un proceso inverso al ejemplo de "la boda de la asistenta" con su empleador, quien, realizando las mismas tareas, baja el PIB porque deja de cobrar un sueldo, la implantación de servicios públicos de ayuda a domicilio, centros de día, residencias etc, aumentaría el PIB al crear empleos y sueldos donde antes sólo había cuidados familiares no pagados, que la exposición de motivos de la Ley reconoce insostenibles por el aumento de la longevidad, la reducción de las familias y la igualdad de las mujeres. La memoria económica de la Ley de Dependencia cifraba en un 3\% ese incremento del PIB (unos 32.000 millones de $€$, de ellos el $66 \%$ de financiación pública), y en 600.000 los empleos a crear. Cinco años después, el informe al Consejo de Ministros de 4.11.11 estimaba que el gasto público en atención a la dependencia se duplicó de 2003 a 2010 -del $0,32 \%$ al $0,64 \%$ del PIB-, pero revelaba datos de su deficiente aplicación por insuficiente financiación, ya que: sólo se crearon 165.000 empleos; sólo se atendió, y tarde, a 760.000 personas y prestación económica, la llamada "paguita" a la cuidadora familiar no fue excepcional, como dice el artículo 18 de la Ley, sino la prestación mayoritaria (51\% en 2009). Desde entonces, aunque es positivo que el peso de la paguita $^{23}$ haya bajado al $42,4 \%$, su desprotección de Seguridad Social la precariza aún más. La financiación estatal ha disminuido en un 23\% desde 2011 (de 1.567 millones a 1.206 millones de euros), y sólo cubre el 19,7\% del coste. En vez de avanzar hacia la suficiencia y cobertura universal, el número de beneficiarios ha bajado de 738.587 en diciembre 2011 a 724.823 en julio 2014, produciendo una refamiliarización y re-feminización del cuidado de las personas dependientes.

Como se plantea en el manifiesto de cien organizaciones feministas ente los Presupuestos del Estado para $2014^{24}$, no podemos absorber la deuda de los bancos con decenas de miles de millones de recortes en sanidad, educación, pensiones y servicios sociales, ni compensarlos aumentando silenciosamente la carga de cuidados. Las feministas y todas las mujeres tenemos intereses para demandar y participar en una auditoría ciudadana de la deuda.

\footnotetext{
${ }^{23}$ Ver Atención a la dependencia: servicios públicos versus "cuidadoras familiares” en pág. 207 y ss de Desiguales por ley, las políticas públicas contra la igualdad de género. María Pazos 2013.

${ }^{24}$ http://impactodegeneroya.blogia.com/ Es el sexto año en que se presenta este análisis feminista del Proyecto de Presupuestos, crítico con el Informe Oficial de Impacto de Genero que los acompañan también desde 2008, después de un recurso contencioso de organizaciones feministas.
} 


\section{ALTERNATIVAS FEMINISTAS: ¿REFORMA O REVOLUCIÓN? ¿DEFENDER LO PÚBLICO O LO COMUNITARIO? ¿ESTADO DE BIENESTAR?}

Estas reivindicaciones de empleo, de políticas públicas de educación, sanidad, pensiones y atención a la dependencia universales, características del Estado de Bienestar, así como de políticas de igualdad de género, se realizan por una parte significativa del movimiento feminista, que participan en actividades como la Plataforma CEDAW Sombra o el Manifiesto ante los Presupuestos y los decretos-leyes. Pero otro importante sector del movimiento cuestiona "la estrategia de emancipación a través del empleo" y afirma: "frente a la crisis no queremos empleo, no queremos salario, no queremos Estado del bienestar. Queremos cuestionar la relación salarial misma, la estructura capitalista en su conjunto" (Pérez Orozco, 2014: 53$)^{25}$. Parte de la polémica intrafeminista en nuestro país puede expresarse en los términos de la polémica Fraser-Butler ${ }^{26}$ sobre reparto y/o reconocimiento, trabajo y/o sexualidad, aunque nuestra sociedad, nuestra política y los movimientos sociales hegemónicos no son los de Estados Unidos, y menos en este periodo de crisis económica y política. El Anteproyecto de recorte aborto, aprobado por el Gobierno en diciembre de 2013 y retirado en septiembre de 2014 ha concitado las mayores movilizaciones feministas en España en décadas, gracias a que la idea feminista de que la maternidad es una decisión de la mujer ha calado como nunca antes. En los numerosos debates sobre si el movimiento feminista puede sumar las mismas fuerzas y tener la misma presencia social más allá del aborto, por encima de que tengamos biografías, acciones, organizaciones o corrientes feministas, referencias, alianzas e interlocuciones políticas diferentes, dudamos de que pueda darse a corto plazo. Ni siquiera hay un debate significativo sobre los cambios impuestos a las madres presentes y futuras por la reducción del

25 Amaia Pérez Orozco, "Subversión feminista de la economía" 2014, página 53. Aunque, respecto al empleo, en ese mismo párrafo matiza "Se trata de preguntarnos no sólo como lograr trabajo para todxs, sino para qué trabajamos (...) El reto es definir democráticamente a qué vamos a llamar buen vivir y cómo vamos a convertirlo en responsabilidad colectiva", y en pags. 263 y 264 considera posible estar "en defensa de lo público, contra el Estado del bienestar" que rechaza por sostenerse "sobre la depredación medioambiental, el expolio de los países de la periferia y la división sexual del trabajo".

${ }^{26}$ En el debate entre ambas profesoras universitarias norteamericanas Nancy Fraser (ver $L a$ politica feminista en la era del reconocimiento: un enfoque bidimensional de la justicia de género; Arenal, jul-dic 2012) cree que el desplazamiento del feminismo de las teorías cuasimarxistas, centradas en el trabajo y la redistribución, a las teorías "post-marxistas" de la cultura, la identidad y el reconocimiento, hace el juego al poder neoliberal, mientras Judith Butler, líder de la teoría queer, considera (ver El marxismo y lo meramente cultural) que la sexualidad está en la estructura política fundamental, sin distinción entre vida cultural y material. 
empleo, de la protección social y de los servicios. Sin embargo, el entramado político es tan fluido desde la irrupción de Podemos - cuyo punto 5.2.2 de Un proyecto económico para la gente defiende políticas de igualdad de género, universalización de la escolarización infantil de 0 a 3 años y de la atención pública a las personas en situación de dependencia, permisos iguales por maternidad y paternidad, etc.-, que posiciones antes irreconciliables, por ejemplo respecto a votar, a presentarse a las elecciones, a hacer seguimiento de las instituciones y las políticas públicas, están cambiando. El zarpazo neoliberal no es pasajero, y es imprescindible que el conjunto del movimiento feminista construyamos un discurso común de cómo afecta a las mujeres y de acciones comunes para evitarlo.

Pero, además de la exacerbación de la desigualdad económica a nivel estatal, europeo y mundial, el cambio climático y el agotamiento medioambiental son otro gran obstáculo a la universalidad de los derechos humanos, cuya superación exige también la modificación urgente de los modelos de producción y consumo. El Informe de la ONU para la Asamblea Cairo +20 sobre población y desarrollo en 2014 alerta sobre la superación en 2013, por primera vez en 3 millones de años, de la temida cota de 400 partes por millón de $\mathrm{CO}^{2}$ en la atmósfera, y los Objetivos para el Desarrollo Sostenible post 2015, en los que el encaje de la igualdad de género a mi juicio es débil, se plantean bajo el lema "una agenda para erradicar antes de 2030 la pobreza, lograr la prosperidad compartida y la paz, y proteger el planeta sin dejar a nadie atrás".

\section{CONCLUSIONES.}

1. La ofensiva neoliberal ha transformado la economía, agrandando las desigualdades, y la oposición a sus políticas ha generado un nuevo marco político. La desigualdad de género no es visible en la nueva agenda política. El movimiento feminista tiene que conquistar de nuevo su espacio político.

2. No hay política feminista sin incidencia en los poderes que influyen en la sociedad, para la que es necesaria la movilización, pero también una interlocución con las instituciones democráticas, ejercer activamente la ciudadanía participativa como organizaciones feministas.

3. Naciones Unidas es, comparada con España y con la Unión Europea, el ámbito institucional más abierto a la voz crítica de las organizaciones no gubernamentales de mujeres. Pero incidir eficazmente en su agenda actual y post 2015 exige sumar fuerzas intrafeministas y dedicar fuerzas a construir alianzas de aquí y a nivel europeo e internacional con otros movimientos, como las mareas en defensa de los bienes públicos universales, el 
movimiento sindical, para la defensa desde abajo del empleo digno y los derechos sociales, los movimientos de regeneración democrática, que sacuden un planteamiento simplista de democracia paritaria de sexos, y el movimiento ecologista, en un intercambio real de visiones y tareas.

4. Siendo necesarias políticas mundiales para los problemas mundiales, necesitamos también la ciudadanía activa como feministas en nuestro país, máxime en 2015, con elecciones locales, autonómicas y generales, resituando la autonomía y la unidad de acción.

5. El movimiento feminista, en su pluralidad, y administrando su curriculum y herencia centenarias, entra en un nuevo ciclo político, que requiere en primer lugar un nuevo programa reivindicativo, del que ha de formar parte importante un marco económico para la igualdad, nuevas o renovadas organizaciones con relevo generacional y medios humanos y materiales que no dependan de líneas de subvención fagocitadoras.

Todo esto es posible, porque en parte ya está pasando. Pero ojalá tengamos inteligencia, fuerza y solidaridad para estar a la altura de las desigualdades y sufrimientos que nos sublevan.

\section{BIBLIOGRAFÍA}

ANGULO, Carlos y HERNÁNDEZ, Sara (2014): Valoración del trabajo doméstico en 2010 y su comparación con la de 2003. Estimación de la serie 2003-2010.

Otras facetas de la Encuesta de Empleo del Tiempo 2009-2010. Madrid: INE.

BUTLER, Judith (2000) El marxismo y lo meramente cultura. New Left Review $\mathrm{N}^{\circ} 2$. Buenos Aires: Ediciones Herramienta.

ESQUIVEL, Valeria y otras (2012): La Economía Feminista desde América Latina. Una hoja de ruta sobre los debates actuales en la región. Santo Domingo: ONU Mujeres.

FACIO, Alda (2001): Cómo hacer Informes paralelos a la CEDAW. Costa Rica: ILANUD.

FRASER, Nancy (2012): La política feminista en la era del reconocimiento: un enfoque bidimensional de la justicia de género. Arenal. Revista de Historia de las Mujeres. Volumen 19, $\mathrm{n}^{\mathrm{0}}$ 2. Instituto de Estudios de la Mujer. Universidad de Granada.

NACIONES UNIDAS, IV Conferencia mundial sobre las mujeres. Declaración de Beijing y Plataforma para la Acción. Septiembre 1995 (1996) Madrid: Ministerio de Asuntos Sociales, Instituto de la Mujer.

NACIONES UNIDAS, Asamblea General (2014): A/69/700 El camino hacia la dignidad para 2030: acabar con la pobreza y transformar vidas protegiendo el 
planeta. Informe de sintesis del Secretario General sobre la agenda de desarrollo sostenible después de 2015.

PAZOS, María (2013): Desiguales por ley, las políticas públicas contra la igualdad de género.

PÉREZ OROZCO, Amaia (2014): Subversión feminista de la economía. Madrid: Traficantes de sueños.

SAN JOSÉ SERRAN, Begoña (2011): No solo queremos cuidar, ni queremos hacerlo solas. Apuntes Ciudadanos 4: 157-174. Madrid, Federación Regional de Asociaciones de Vecinos.

VVAA (2012): 25 años del Fórum de Política Feminista y de feminismo 1987-2012 ¿Abriendo nuevo ciclo? Madrid: Fórum de Política Feminista.

VVAA (2014): Feminismo frente al neoliberalismo: estrategias y alianzas. Madrid: Fórum de Política Feminista.

VVAA (2011): Estudio sobre la aplicación de la Ley Orgánica 3/2007, de 22 de marzo, para la Igualdad Efectiva de Mujeres y Hombres. Barcelona: Generalitat de Catalunya. Centre d'Estudis Juridics i Formació Especialitzada. 